\title{
SIMULAÇÃO DA OPERAÇÃO DE FRATURAMENTO HIDRÁULICO EM FORMAÇÕES DE XISTO
}

\author{
SIMULATION OF THE HYDRAULIC FRACTURING OPERATION IN SHALE GAS \\ FORMATIONS
}

\author{
Daniele Amaral OLIVEIRA; Sérgio Augusto B. FONTOURA \\ Pontifícia Universidade Católica do Rio de Janeiro. Departamento de Engenharia Civil. Rua Marquês de São Vicente, 225 - Gávea, \\ Rio de Janeiro - RJ. E-mails: danieleamaraldeoliveira@gmail.com; fontoura@puc-rio.br

Introdução
Definição do Problema
Metodologia
Discussões
Conclusão
Agradecimentos
Referências

RESUMO - Atualmente a produção de gás a partir de formações ricas em xisto é uma das áreas de maior expansão na exploração de petróleo. Devido à permeabilidade extremamente baixa para se conseguir sucesso neste tipo de formação é fundamental a utilização de poços horizontais associados à técnica de fraturamento hidráulico, esta que por sua vez visa aumentar a produção de gás, conectando uma rede de fraturas ao poço. Porém, o caminho da fratura hidráulica e quais os parâmetros que influenciam nessa operação ainda são foco de discursão na comunidade geotécnica. Logo este estudo consiste na construção de um modelo numérico num software de elementos finitos, para análise de inicialização e propagação de fraturas hidráulicas em formações de baixa permeabilidade e impermeáveis, com objetivo de expor os fatores que governam a operação de fraturamento hidráulico.

Palavras-chave: Elementos finitos, fraturamento hidráulico, Shale gas.

ABSTRACT - Currently the production of gas from shale-rich formations is one of the areas of greatest expansion in oil exploration. Due to the extremely low permeability to achieve success in this type of formation, it is essential to use horizontal wells associated with the hydraulic fracturing technique, which in turn aims to increase gas production by connecting a network of fractures to the well. However, the hydraulic fracture path and the parameters that influence this operation are still the focus of discourse in the geotechnical community. This study consists in the construction of a numerical model in a finite element software for initialization and propagation analysis of hydraulic fractures in low permeability and impermeable formations, aiming at exposing the factors that govern the hydraulic fracturing operation.

Keywords: Finite elements, hydraulic fracturing, Shale gas.

\section{INTRODUÇÃO}

O fraturamento hidráulico é uma técnica de estimulação de poços muito comum e importante para melhorar o índice de produção de poços na indústria de petróleo e gás.

É basicamente um processo de iniciar e propagar uma trinca, através da pressurização de um fluido no poço, desta forma, cria-se um canal de alta condutividade, para que os hidrocarbonetos fluam para o poço, aumentando assim a taxa de produção e $\mathrm{o}$ indice de recuperação (Fjaer, 2008).

Esta técnica foi utilizada pela primeira vez em 1903 (Economides, 2000), porém o uso comercial só ocorreu em 1948 (Frydman, 1997. Inicialmente, começou apenas como tratamento de estimulação para recuperação secundária dos poços de a exploração e produção sendo utilizado principalmente em reservatórios com permeabilidade de moderada a alta.

No entanto, com o tempo as empresas petrolíferas começaram explorar em formações com baixa permeabilidade, desta forma, o fraturamento hidraúlico tornou-se um projeto de tratamento para recuperação primária.

Especialmente hoje, com o aumento da atividade de perfuração em reservatórios não convencionais de gás em xisto, arenidos fechados, e gás de carvão, onde é conhecido que a permeabilidade é geralmente muito baixa na escala de nanodarcies, sendo assim a a prudução desses reservatórios sem um tratamento de freturamento hidráulico é inviável.

Os mecanismos de produção e as características do reservatório para esses reservatórios podem variar muito dependendo da geologia regional e do ambiente deposicional. Sendo assim, é - um vasto estudo da área a ser estimulada.

Existem diversos simuladores de fratura, baseados em elementos finitos (Lam et al., 1986; Boone \& Ingraffea, 1990; Papanastasiou. 1997; 
Frydman, 1997) e de contorno (Clifton \& AbouSayed, 1979; Yew \& Liu, 1993; Yamamoto et al., 1999; Rungamornrat et al., 2005) que preveem a geometria da fratura hidráulica.

Porém poucos preveem a fratura considerando a permeabilidade da formação, desta forma, este estudo visa analisar a influência da permeabilidade na propagação de fratura hidráulica, pois formações de xisto são conhecidas pela baixíssima permeabilidade o que pode influenciar consideravelmente na geometria da fratura hidráulica.

O estudo trata-se da construção de um modelo em um programa de elementos finitos, sendo considerada uma formação de baixíssima permeabilidade, e depois é realizada uma análise de sensibilidade com a permeabilidade para que se possa considerar a influência desta na geometria da fratura e nos outros parâmetros fundamentais ao sucesso da operação de fraturamento hidráulico como método de recuperação de hidrocarbonetos.

\section{DEFINIÇÃO DO PROBLEMA}

A figura 1 trata-se de um modelo que consiste em uma fatia circular com $1 \mathrm{~m}$ de espessura de rocha contendo óleo com uma pequena rachadura inicial modelada.

O domínio tem um diâmetro de 160 m. Devido à simetria apenas metade do diâmetro é modelado. As tensões in situ utilizadas são: $8,55 \mathrm{MPa}$, $12,55 \mathrm{MPa}, 15 \mathrm{MPa}$. O modelo é analisado em duas etapas: um passo geostático, onde se aplica uma pressão de poros na formação e as tensões in situ, após o equilíbrio de a formação ser atingindo, uma segunda etapa de fraturamento hidráulico começa, onde é injetado um fluido a uma taxa de $5 \times 10^{6} \mathrm{~m}^{3} / \mathrm{s}$. O tempo de duração da etapa de injeção é de 160 s. O quadro 1 apresenta os dados utilizados na modelagem.

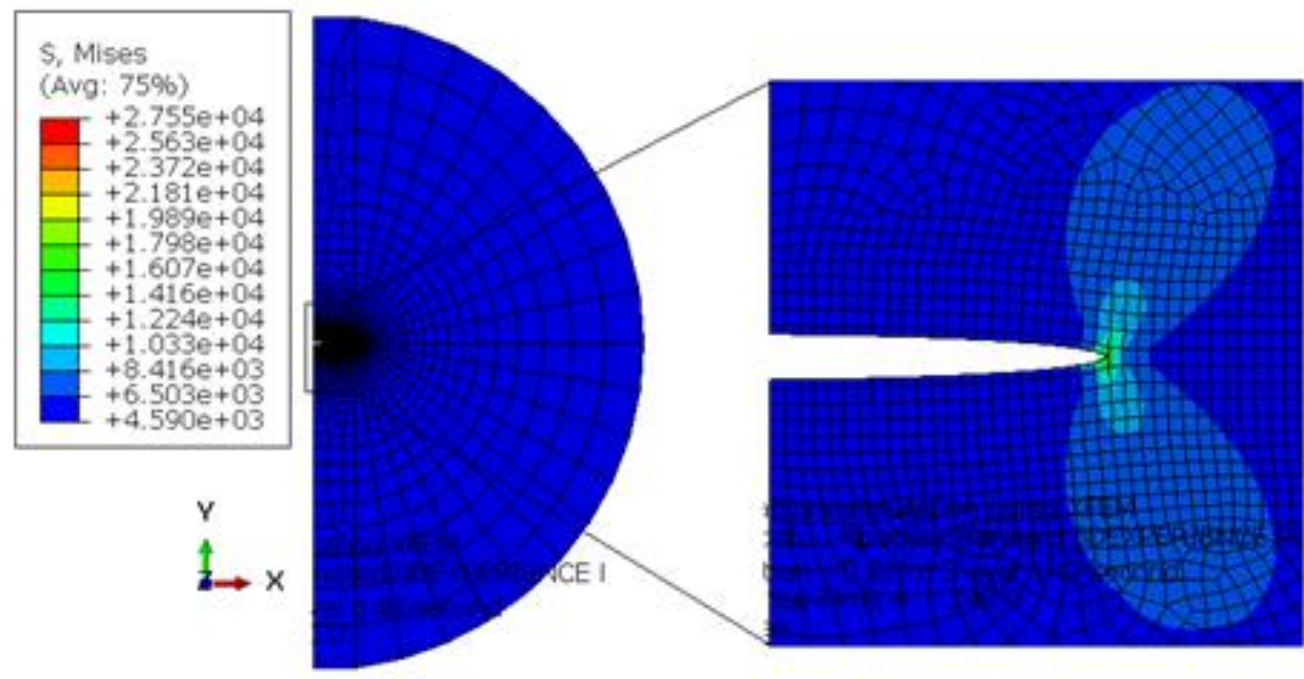

Figura 1 - Região do poço de petróleo fraturado.

Tabela 1 - Propriedades Geomecânicas utilizadas na simulação

\begin{tabular}{c|c|c}
\hline Parâmetro & Magnitude & Unidade \\
\hline Módulo de Elasticidade & $1,294 \mathrm{e}+06$ & $\mathrm{MPa}$ \\
\hline Coeficiente de Poisson & 0,25 & $\mathrm{ADM}$ \\
\hline Porosidade & 0,25 & $\mathrm{~m} / \mathrm{s}$ \\
\hline Condutividade hidráulica & $2,418 \times 10^{-8}$ & $\mathrm{kPaS}$ \\
\hline Fluido saturante & $1 \times 10^{-6}$ & $\mathrm{kPa} / \mathrm{m}$ \\
\hline \multicolumn{2}{c}{ Fluido Fraturante } \\
\hline Peso específico do fluido & 9.8 & $\mathrm{kPa} . \mathrm{s}$ \\
\hline Viscosidade & $1 \times 10^{-6}$ & $\mathrm{~N} / \mathrm{m}$ \\
\hline \multicolumn{2}{c}{ Fratura } & $\mathrm{MPa}$ \\
\hline Energia da Fratura & $28 \times 10^{3}$ & $\mathrm{MPa}$ \\
\hline Fator Intensidade de Tensão & $8,5 \times 10^{4}$ & $\mathrm{~m} /(\mathrm{kPa} \mathrm{s})$ \\
\hline Resistência a tração & 0,32 & $\mathrm{n} 4 \mathrm{n}$ \\
\hline Leakoff & $5,879 \times 10^{-10}$ & \multicolumn{2}{c}{} \\
\hline
\end{tabular}




\section{METODOLOGIA}

O comportamento considerado nas fraturas é o de coesão na ponta da trinca, onde foi adotado como critério de falha a tensão principal máxima, para determinar o início do dano do material. A lei de evolução do dano é baseada no critério de energia, e tem por base a lei de BK (Benzeggagh
\& Kenane, 2006), está por sua vez é responsável pela propagação do dano ao longo dos elementos enriquecido.

Onde $G$ está relacionado ao modo e fraturamento. As modos de fraturamento são descritos na figura 2 .

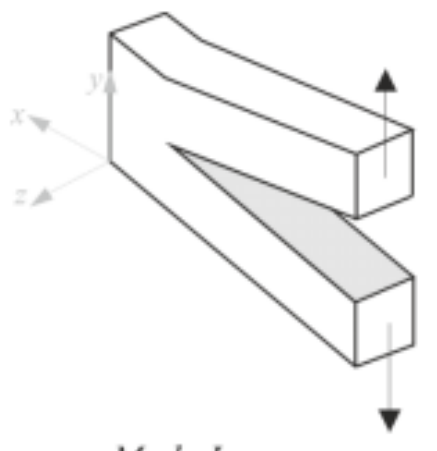

Modo I

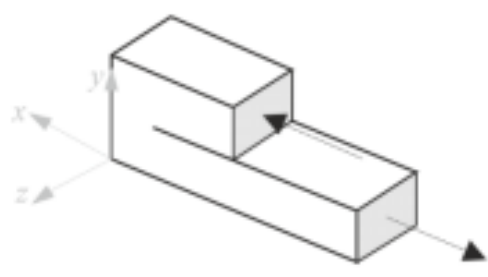

Modo II

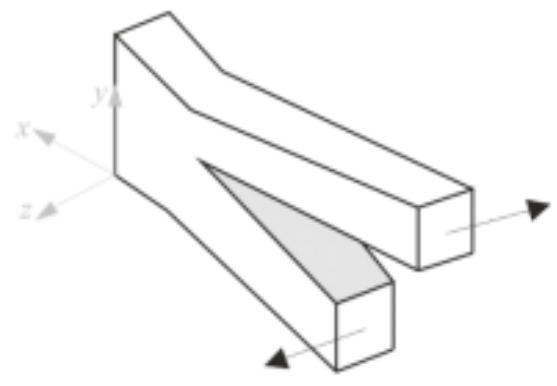

Modo III

Figura 2 - Modos de propagação da fratura (Frydman, 2006).

Reeder \& Creews (1990) demostraram que, para gerar dados experimentais nos modos mistos de fratura, era necessária uma extensa campanha de laboratório. Dessa forma, desenvolveram critérios empíricos utilizados no cálculo da resistência à fratura para modos mistos.

O programa Abaqus dispõe destas soluções para a modelagem numérica de fraturas via XFEM (Dassault Systèmes, 2013), entre elas: a lei de Potência, a lei BK e a Lei de Reeder. A lei de potência é dada pela equação 1 .

A lei de potência é uma expressão matemática empírica que representa as diferentes respostas dos materiais, onde o comportamento específico é idealizado, alterando os valores atribuídos dos parâmetros de ajuste, $\alpha, \beta, \gamma$. Porém, para a seleção desses parâmetros deve-se realizar alguns ensaios de modo misto.

Uma vez determinada à tenacidade à fratura do material $(G c)$, e utilizando os conceitos da Mecânica da Fratura, estabelece-se um critério de início e propagação das trincas mediante a comparação de $G_{C}$ com a taxa de liberação de energia $(G)$. O início da fratura é dado quando $G>G c$.

$$
\left(\frac{G_{I}}{G_{l c}}\right)^{\alpha}+\left(\frac{G_{I I}}{G_{l l c}}\right)^{\alpha \beta}+\left(\frac{G_{I I I}}{G_{I I I C}}\right)^{\gamma}=1
$$

\section{DISCUSSÕES}

A figura 3 apresenta a abertura da fratura, numa distância de até 4,5 metros distante do poço, primeiro foi analisado o modelo base com os dados inicias fornecido no quadro acima. Para em seguida ser feita a análise de influência da permeabilidade na fratura.

$\mathrm{Na}$ figura 4 é possível observar que quanto menor a condutividade hidráulica do material circundante, maior a abertura da fratura. Isto é devido ao fato de menos fluido é perdido para a formação, porém como a vazão foi mantida observamos um encurtamento da fratura hidráulica.

Pois o mesmo volume de fluido foi injetado em todos os casos, para que fosse possível uma análise mais criteriosa dos parâmetros envolvidos na simulação.

A figura 5 apresenta o resultado da taxa de leakoff o programa apresenta boa concordância entre as curvas e é possível notar o efeito de sucção na ponta da fratura.

A figura 6 mostra a pressão liquida ao longo da fratura e a pressão de injeção. É possível ver que quanto menor a condutividade da formação maior a pressão liquida ao longo da fratura, este fato vai de acordo com os resultados de geometria da fratura, visto que, é possível observar uma maior abertura da fratura também.

Já na pressão de injeção, quanto menor a condutividade maior a pressão de injeção requerida para fraturar a formação, visto que o excesso de poro pressão será menor. 


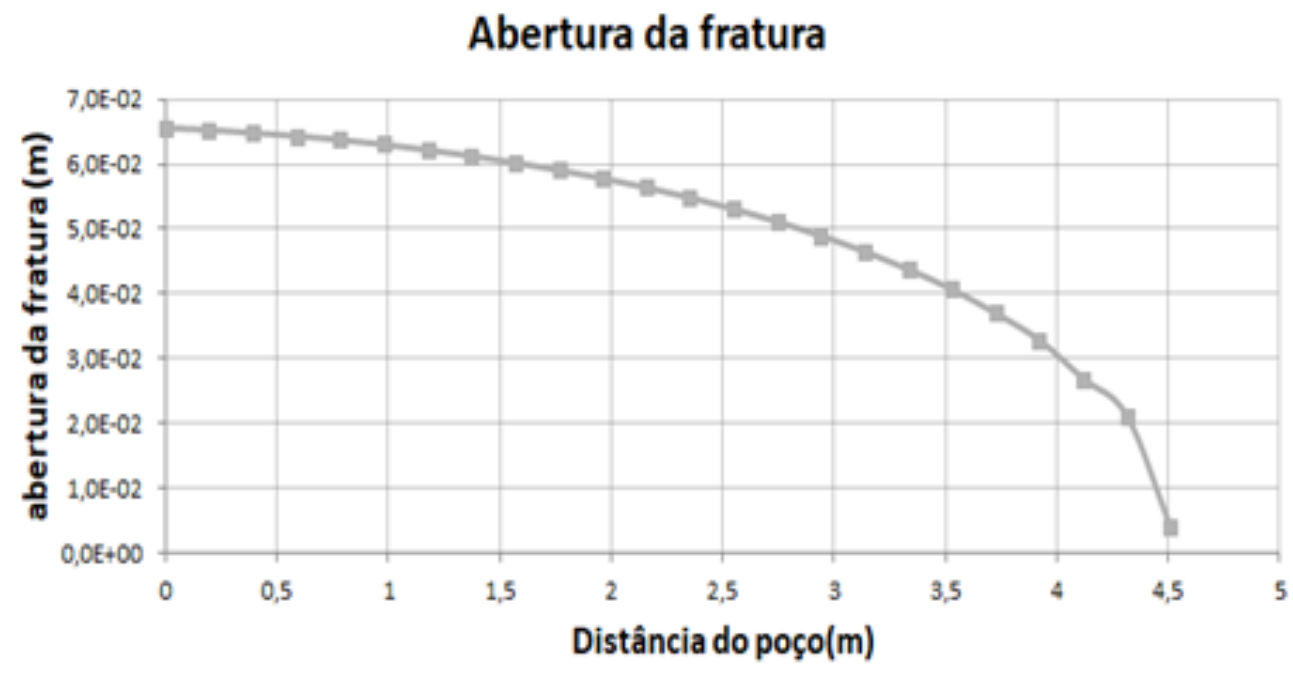

- abertura da fratura

Figura 3 - Abertura e comprimento da fratura.

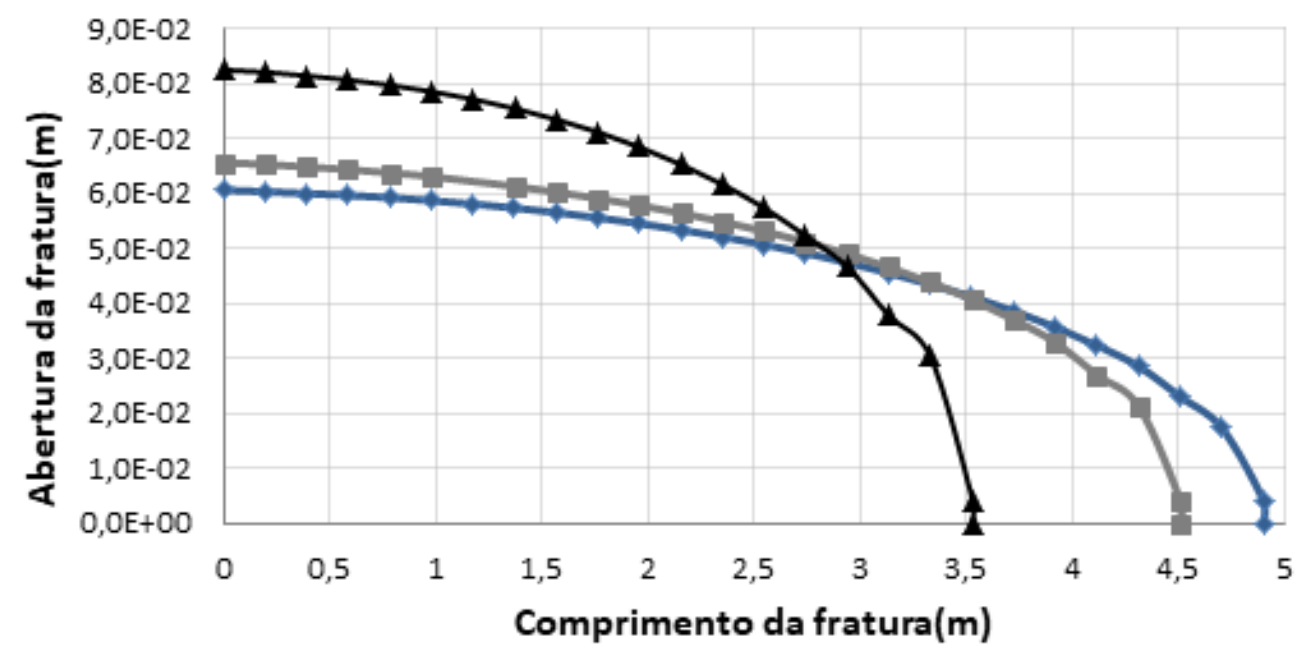

$\longrightarrow \mathrm{K}=10^{\wedge}-7 \mathrm{~m} / \mathrm{s} \rightarrow \mathrm{K}=10^{\wedge}-8 \mathrm{~m} / \mathrm{s} \rightarrow \mathrm{K}=10^{\wedge}-9 \mathrm{~m} / \mathrm{s}$

Figura 4 - Influência da permeabilidade na geometria da fratura.

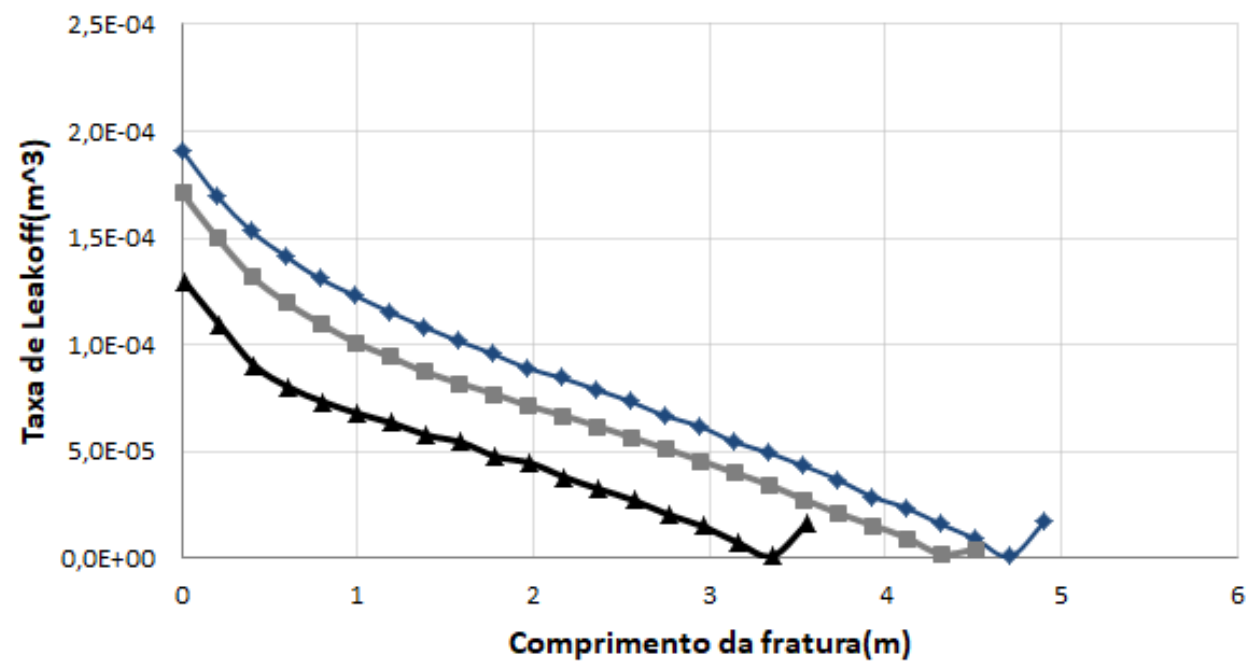

$\longrightarrow K=10^{\wedge}-7-K=10^{\wedge} 10-8 \rightarrow k=10^{\wedge}-9$

Figura 5 - Influência da permeabilidade na taxa de Leak-okk. 


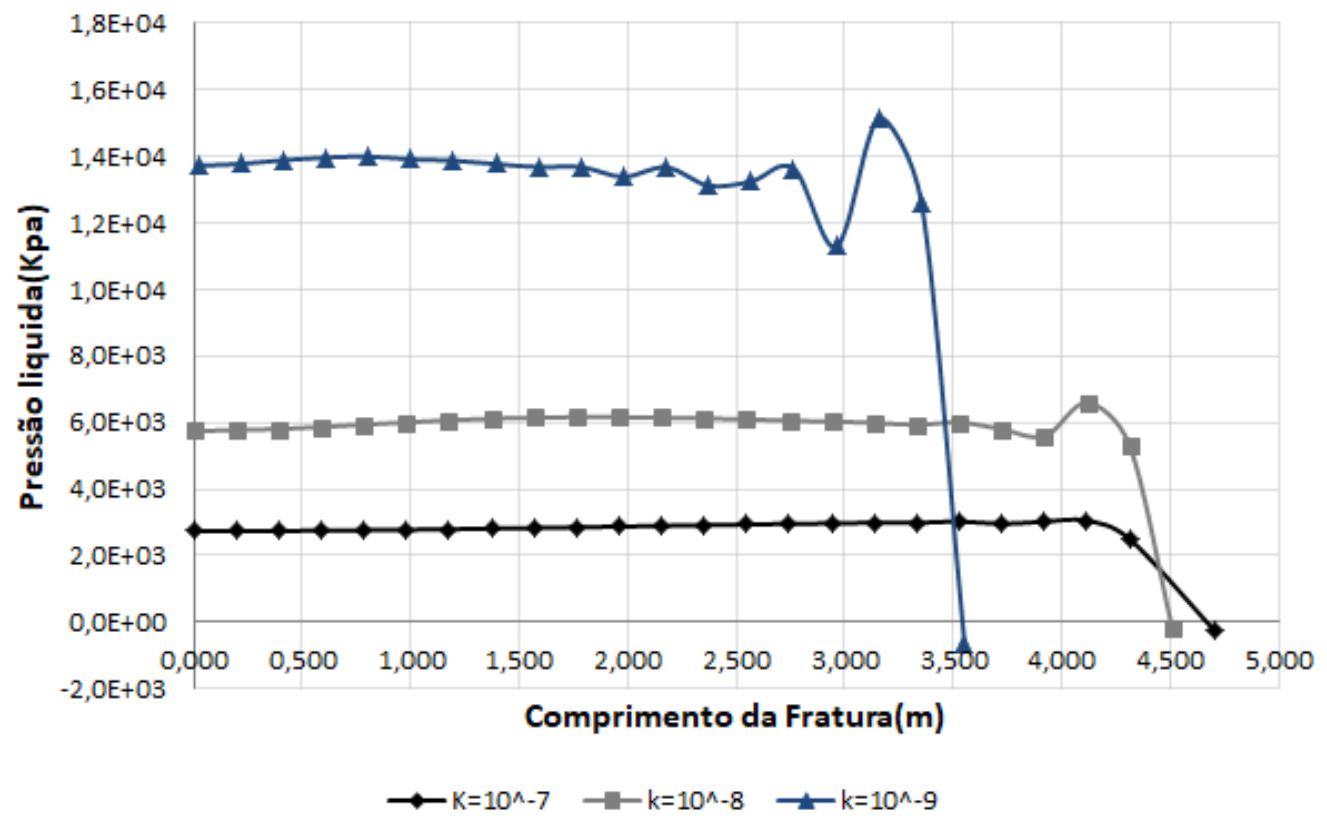

Figura 6 - Influência da permeabilidade na pressão liquida.

\section{CONCLUSÕES}

Reservatórios de xisto são atualmente de grande interesse a matriz energética mundial, visto a sua grande capacidade de produzir gás, sendo este um dos principais combustíveis e o de maior expansão de consumo. Desta forma, é essencial o estudo de técnicas capazes de aumentar a recuperação de hidrocarbonetos nestes reservatórios ditos não convencionais. $\mathrm{O}$ fraturamento hidráulico é um método essencial de estimulação em reservatórios não convencionais. Porém, sobre configurações geológicas complexas, é extremamente difícil controlar a evolução da fratura hidráulica. Todavia esta operação é de grande risco operacional, visto que, a fratura pode atravessar diversas formações e ocasionar vazamentos. Assim, é fundamental o entendimento dos fatores que influenciam na geometria final da fratura, em relação as condições de tensão in situ da formação.

Em decorrência da baixíssima permeabilidade do xisto, estes reservatórios só produzem com alguma técnica de estimulação e desta forma, o fraturamento hidráulico é usado para criar canais de alta condutividade e interconectar as fraturas naturais existente na formação. No entanto, um dos fatores que mais influenciam nesta operação é a permeabilidade e a porosidade da formação.
Conseguimos concluir neste estudo, que quanto menor a permeabilidade da formação, maior será a abertura da fratura, visto que as formações de xisto em muitos lugares ao redor do mundo são laminações de baixa espessura. Este fato deixa como experiência que a abertura deve ser controlada de forma a não fraturar formações adjacentes, que poderão ocasionar quebra da integridade do selo. É importante salientar que, muitas vezes o objetivo da operação é criar fraturas de grande extensão para interconectar a rede de fraturas pré-existente na formação. Sendo assim, torna se fundamental o aumento da permeabilidade da formação, que pode ser feito com injeção de fluidos previamente aquecidos.

Este estudo manteve o foco apenas na permeabilidade da formação, porém muitas são as incógnitas que influenciam na operação de fraturamento hidráulico. As limitações do conhecimento dos mecanismos que influenciam a fratura hidráulica, em formações não convencionais, vêm restringindo o uso de técnicas inovadoras de estimulação de poços, sendo assim, todo esforço para desvendar tais mecanismos e para confirmar o que já vem sendo exposto na literatura é de grande importância ao desenvolvimento sustentável dos campos de gás em folhelho.

\section{AGRADECIMENTOS}

Agradecimento ao Programa de Pós-Graduação em Engenharia Civil - Departamento de Engenharia Civil- PUC -RIO, aos colegas do grupo de Tecnologia de engenharia de Petróleo- GTEP e à Coordenação de Aperfeiçoamento de Pessoal de Nível Superior-CAPES. 


\section{REFERÊNCIAS}

BENZEGGAGH, M. \& KENANE, M. "Measurement of Mixed-Mode Delamination Fracture Toughness of Unidirectional Glass/Epoxy Composites with MixedMode Bending Apparatus," Composite Science and Technology, v. 56, p. 439, 1996.

BOONE, T.J. \& INGRAFFEA, A.R. A numerical procedure for simulation of hydraulically-driven fracture propagation in poroelastic media. Int. J. Num. and Anal. Methods in Geomechanics, v. 14, n. 1, p. 27-47, 1990. doi:10.1002/ nag.1610140103.

CLIFTON, R.J. \& ABOU-SAYED, A.S. On the Computation of the Three-Dimensional Geometry of Hydraulic Fractures. In: SYMPOSIUM ON LOW PERMEABILITY GAS RESERVOIRS. Denver, 1979. Paper SPE 7943, Denver, 1979. doi: 10.2118/7943-MS.

ECONOMIDES, M., NOLTE, K.,. Reservoir Stimulation, 3rd ed. John Wiley \& Sons, Chichester, UK.,2000.

FJAER. R. M.; HORSRUD, A.; RAAEN, A.M.; RISNES, R.; R.M. Petroleum related rocks mechanics. 2 ed., 337 p., 2008

FRYDMAN, M. Iniciação e Propagação de Fraturas em Poços de Petróleo. Rio de Janeiro. 1996. Tese (Doutorado), PUC-Rio,

LAM, K.Y.; CLEARY, M.P.; BARR, D.T. A Complete Three-Dimensional Simulator for Analysis and Design of Hydraulic Fracturing. In: UNCONVENTIONAL GAS TECHNOLOGY SYMPOSIUM, 1986, Kentucky. Paper SPE 15266, Kentucky. doi:10.2118/15266-MS.

PAPANASTASIOU, P.C. A coupled elastoplastic hydraulic fracturing model. International Journal of Rock Mechanics and Mining Science, v. 34, n. 3-4, 240.e1-240.e15, 1997. doi:10.1016/S1365 1609(97)00132-9.
REEDER, J.; S.KYONGCHAN, P.B. In: Chunchu, \& Ambur, D.R. (eds) "Postbuckling and Growth of Delaminations in Composite Plates Subjected to Axial Compression"43rd AIAA/ASME/ASCE/AHS/ASC Structures. In: STRUCTURAL DYNAMICS, AND MATERIALS CONFERENCE, Denver, 2002. Actas... Denver, v. 1746, p. 10.

RUNGAMORNRAT, J., WHEELER, M.F.; MEAR, M.E. A Numerical Technique for Simulating Nonplanar Evolution of Hydraulic Fractures. In: SPE ANNUAL TECHNICAL CONFERENCE AND EXHIBITION, Dallas, 2005. Paper SPE 96968. Dallas. doi: 10.2118/96968-MS.

YAMAMOTO, K., SHIMAMOTO, T.; MAEZUMI, S. Development of a True 3D Hydraulic Fracturing Simulator. In: SPE ASIA PACIFIC OIL AND GAS CONFERENCE AND EXHIBITION, 1999. Jakarta. Paper SPE 54265 Jakarta, 1999. doi: 10.2118/54265MS.

YEW, C.H. \& LIU, G.F. Fracture Tip and Critical Stress Intensity Factor of a Hydraulically Induced Fracture. SPE Prod \& Fac, v. 8, n. 3, p. 171-177, 1993. SPE22875-PA. doi: 10.2118/22875-PA

Submetido em 16 de janeiro de 2019 Aceito em 18 de dezembro de 2019 